\title{
On an Abstract Analog of the Bellman-Gronwall Inequality
}

\author{
By
}

D.D. BAINOV*, A.D. MYSHKIS** and A.I. ZAHARIEV*

\begin{abstract}
The paper considers one of the possible generalizations of the Volterra equations in the case when the independent variable belongs to an arbitrary compact metric space and, as a corollary of the obtained results, an abstract analog of the Bellman-Gronwall inequality is proved.
\end{abstract}

Recently there has been a considerable interest in integral inequalities for scalar functions of a vector argument and now many papers deal with that subject. Extensive reference is given in [1], [2]. The integral domain in the above mentioned works is an $n$-dimensional parallelepiped (see for instance [3]). In the monograph [4] an analog of the Bellman-Gronwall inequality for precompact domains in $\mathbb{R}^{n}$ is proved, which, in general, are not a Cartesian product of intervals.

The aim of the present paper is to prove the Bellman-Gronwall inequality in the case of a compact metric space.

Let $\Omega$ be a compact metric space with a metric $\rho$ and a Borel measure $\mu$, and let for each $x \in \Omega$ the mapping $M: x \rightarrow M_{x}$ be defined where $M_{x}$ is a closed subset of $\Omega$. We shall suppose that the mapping $M$ satisfies the following condition:

A1. For each $\varepsilon>0$ and each $x \in \Omega$ there exists a number $\delta>0$ such that for each $y \in \Omega$, for which $\rho(x, y)<\delta$ the following inequality holds

$$
\mu\left(\left\{M_{x} \backslash M_{y}\right\} \cup\left\{M_{y} \backslash M_{x}\right\}\right)<\varepsilon .
$$

Defimition $\mathbb{H}_{\text {. The }}$ mapping $M$ is said to be continuous with respect to the measure $\mu$ if it satisfies condition $\mathrm{A} 1$.

Communicated by S. Hitotumatu, September 24, 1982.

* University of Plovdiv "P. Hilendarski", Plovdiv, Bulgaria;

** Moscow Institute of Railway Transport Engineers, Moscow, U.S.S.R. 
Consider the equation

$$
\varphi(x)=f(x)+\lambda \int_{M_{x}} K(x, y) \varphi(y) d \mu_{y},
$$

where the kernel $K: \Omega^{2} \rightarrow \mathbb{C}$ and the function $f: \Omega \rightarrow C$ are continuous, and $\lambda \in \mathbb{C}$.

Denote by $C(\Omega)$ the Banach space of the continuous functions $g: \Omega \rightarrow C$ with a norm $\|g\|=\sup _{x \in \Omega}|g(x)|$ and define the linear operator $\mathbb{K}$ by the equality

$$
(\mathbb{K} g)(x):=\int_{M_{x}} K(x, y) g(y) d \mu_{y}, \quad g \in C(\Omega)
$$

The operator $I-\lambda \mathbb{K}, \lambda \in \mathbb{C}, \lambda \neq 0$ is a canonical Fredholm operator. In order to verify the above statement it is sufficient to prove that the operator $\mathbb{K}$ is compact $([5]$, p. 110$)$.

THEOREM 1 . Let the mapping $M$ be continuous with respect to the measure $\mu$. Then the operator maps $C(\Omega)$ into $C(\Omega)$ and is compact.

This theorem is likely to be known but for the sake of completeness we shall give its proof.

Proof. Since $C(\Omega)$ is a Banach space then it is sufficient to show that the image of the unit ball $B \subset C(\Omega)$ is a compact set. For each function $g \in B$, taking into account that $\mu$ is a Borel measure, we obtain

$$
\|\mathbb{K} g\|=\sup _{x \in \Omega}\left|\int_{M_{x}} K(x, y) g(y) d \mu_{y}\right| \leqq A \mu(\Omega),
$$

where $A=\sup _{x, y \in \Omega}|K(x, y)|$, i.e. the norms of the functions belonging to the set $\mathbb{K}(B)$ are uniformly bounded. We shall prove that the set $\mathbb{K}(B)$ is equicontinuous.

Let $\varepsilon>0$ be arbitrary. The uniform continuity of the kernel $K(x, y)$ implies that there exists a number $\delta=\delta(\varepsilon)>0$ such that for arbitrary $x, y, z \in \Omega$ if $\rho(x, y)<\delta$ then

$$
|K(x, z)-K(y, z)|<\frac{\varepsilon}{2 \mu(\Omega)}
$$

Besides, it can be easily verified that the mapping $M$ is uniformly continuous with respect to the measure $\mu$, and hence there exists a number $\delta^{*}>0$, $\delta^{*} \leq \delta$, such that if $\rho(x, y)<\delta^{*}$ then 


$$
\mu\left(\left\{M_{x} \backslash M_{y}\right\} \cup\left\{M_{y} \backslash M_{x}\right\}\right)<\frac{\varepsilon}{2 A \mu(\Omega)} .
$$

Therefore, for each function $g \in B$ and for $x, y \in \Omega$, (3) and (4) yield the estimate

$$
\begin{aligned}
&|(\mathbb{K} g)(x)-(\mathbb{K} g)(y)|=\left|\int_{M_{x}} K(x, z) g(z) d \mu_{z}-\int_{M_{y}} K(y, z) g(z) d \mu_{z}\right| \\
& \leq\left|\int_{M_{x} \cap M_{y}}[K(x, z)-K(y, z)] g(z) d \mu_{z}\right|+\left|\int_{M_{x} \backslash M_{y}} K(x, z) g(z) d \mu_{z}\right| \\
&+\left|\int_{M_{y} \backslash M_{x}} K(y, z) g(z) d \mu_{z}\right|<\varepsilon,
\end{aligned}
$$

which makes it obvious that the functions from $\mathbb{Z}(B)$ are equicontinuous.

Therefore $\mathbb{K}$ maps $C(\Omega)$ into $C(\Omega)$ and it follows from the Ascoli-Arzela theorem that the set $\mathbb{K}(B)$ is compact.

Theorem 1 implies that the Fredholm alternative holds for equation (1).

Suppose in addition that the mapping $M$ satisfies the following conditions:

A2 (Transitivity). For each $x \in \Omega$ and each $y \in M_{x}$ the inclusion $M_{y} \subseteq M_{x}$ holds (in other words $M^{2} \subseteq M$ ).

This enables us to consider, for an arbitrary point $a \in \Omega$ the restriction $\mathbb{K}_{a}: C\left(M_{a}\right) \rightarrow C\left(M_{a}\right)$ of the operator $\mathbb{K}$ where $\mathbb{K}_{a}$ is defined by equality (2). In that case the restriction $\varphi / M_{a}$ of the solution of the equation $\varphi=f+\lambda \mathbb{K} \varphi$, $\varphi \in C(\Omega)$, is a solution of the equation $\widetilde{\varphi}=f / M_{a}+\lambda \mathbb{K}_{a} \tilde{\varphi}, \tilde{\varphi} \in C\left(M_{a}\right)$.

A3 (Semicontinuity from below). For each $x \in \Omega$ and each $\varepsilon>0$ there exists $\delta>0$ such that for each $y \in \Omega$ for which $\rho(x, y)<\delta$ the inclusion $M_{x} \subseteq U\left(M_{y}, \varepsilon\right)$ holds where $U\left(M_{y}, \varepsilon\right)$ denotes the $\varepsilon$-neighbourhood of $M_{y}$.

Remark 1. This condition is very close to condition $\mathrm{A} 1$ and in certain cases is logically related to it. For example, it can easily be verified that if the mapping $M$ satisfies condition A1 then it is semicontinuous from below for every $x \in \Omega$ such that for each $\varepsilon>0$ the inequality

$$
\inf _{y \in \mathbb{H}_{x}}\left[\mu\left(U(y, \varepsilon) \cap M_{x}\right)\right]>0
$$

holds.

A4. There exists $x_{0} \in \Omega$ such that $\mu\left(M_{z_{0}}\right)=0$.

Remark 2. If conditions A1-A4 are fulfilled then equation (1) may be considered as one of the possible generalizations of the Volterra equations. This can be seen, for example, from the fact that if we consider the equation 


$$
\varphi(x)=f(x)+\int_{0}^{\psi(x)} K(x, y) \varphi(y) d y, \quad \psi:[0,1] \rightarrow[0,1]
$$

conditions A1-A4 are fulfilled if and only if the function $\psi(x)$ is continuous and $0 \leq \psi(x) \leq x$ for $x \in[0,1]$. It may be of some interest to describe the structure of the mapping $M$ satisfying conditions $\mathbb{A} 1-\mathbb{A} 4$ in the general case.

Definnition 2. Condition (A) is said to hold if the conditions A1-A4 are fulfilled and $\Omega$ is a connected set.

$\mathbb{T H} \mathbb{H O R \mathbb { R }}$ 2。 Let condition (A) be fulfilled. Then equation (1) has exactly one solution $\varphi \in C(\Omega)$ for each function $f \in C(\Omega)$.

Proof. Consider the equation

$$
\varphi(x)=\lambda \int_{M_{x}} K(x, y) \varphi(y) d \mu_{y}, \quad \lambda \in \mathbb{C}
$$

and let $\varphi \in C(\Omega)$ be one of its solutions. Denote by $H$ the set

$$
H=\left\{x \mid x \in \Omega \text { and for each } y \in M_{x} \text { we have } \varphi(y)=0\right\} .
$$

We shall prove that $H \neq \emptyset$. It follows from $\mathbb{A} 4$ that there exists a point $x_{0} \in \Omega$ such that $\mu\left(M_{x_{0}}\right)=0$ and $M_{x_{0}} \neq \emptyset$ (the case $M_{x_{0}}=\emptyset$ is trivial), and therefore condition $\mathrm{A} 2$ implies that for each $x \in M_{x_{0}}$ we have $\mu\left(M_{x}\right)=0$. Then (5) yields $\varphi(x)=0$ for each $x \in M_{x_{0}}$ and hence $x_{0} \in H$.

The set $\mathbb{H}$ is closed. Let us choose an arbitrary fundamental sequence of points $\left\{x_{n}\right\}, x_{n} \in H, n=1,2, \cdots$ and denote by $x^{*}$ its limit in $\Omega$. We shall prove that $x^{*} \in H$. For this purpose it is sufficient to consider only the case when all $M_{x_{n}}$ and $M_{x^{*}} \neq \emptyset$. Let $z \in M_{x^{*}}$ be an arbitrary point and $\varepsilon>0$ be an arbitrary number. We denote by $y_{n}$ the point at which the minimum of the distance $\rho(z, x), x \in M_{x_{n}}$ is reached, i.e. $\rho\left(z, y_{n}\right)=\rho\left(z, M_{x_{n}}\right)$ (This minimum is reached because $M_{x_{n}}$ are closed sets). There exists a number $\delta_{1}>0$ such that if $\rho(x, z)<\delta_{1}, x \in \Omega$ then $|\varphi(x)-\varphi(z)|<\varepsilon$. Condition $\mathbb{A} 3$ implies that there exists a number $\delta_{2}>0$ such that if $\rho\left(x_{n}, x^{*}\right)<\delta_{2}$, then $M_{x^{*}} \subseteq U\left(M_{x_{n}}, \delta_{1}\right)$. But since $\lim _{n \rightarrow \infty} \rho\left(x_{n}, x^{*}\right)=0$ then there exists a number $N>0$ such that for $n>N$ we have $\rho\left(x_{n}, x^{*}\right)<\delta_{2}$. Therefore for $n>N$ we have $z \in U\left(\mathbb{M}_{x_{n}}, \delta_{1}\right)$. Hence $\rho\left(z, M_{x_{n}}\right)=\rho\left(z, y_{n}\right)<\delta_{1}$ and thus $\left|\varphi(z)-\varphi\left(y_{n}\right)\right|<\varepsilon$. Since $\varphi\left(y_{n}\right)=0$ then $|\varphi(z)|<\varepsilon$ and hence $\varphi(z)=0$. Since $z \in M_{z^{*}}$ is an arbitrary point then $\varphi(x)=0$ for each $x \in M_{x^{*}}$ which implies that $x^{*} \in H$.

We shall prove that $H$ is an open set as well.

Let $a \in H$ be an arbitrary point and let $\varepsilon>0$ be such that the inequality 
$\varepsilon|\lambda| A<\frac{1}{2}$ holds. There exists a number $\delta>0$ such that for each $x \in \Omega$ for which $\rho(a, x)<\delta$, the following inequality holds

$$
\mu\left(\left\{M_{a} \backslash M_{x}\right\} \cup\left\{M_{x} \backslash M_{a}\right\}\right)<\varepsilon 。
$$

Let $b \in \Omega, \rho(a, b)<\delta$ and consider the set

$$
T=\left\{g \mid g \in C\left(M_{b}\right), g(x) \equiv 0 \text { for } x \in M_{b} \cap M_{a}\right\} .
$$

Condition A2 implies that the operator $\lambda \mathbb{K}_{b}$ maps $T$ into $T$. Let $g_{1}, g_{2} \in T$ be arbitrary functions. Then we have

$$
\begin{aligned}
& || \lambda \mathbb{K} g_{1}-\lambda \mathbb{K} g_{2} \|_{M_{b}}=|\lambda| \sup _{x \in M_{b}}\left|\int_{M_{x}} K(x, y)\left(g_{1}(y)-g_{2}(y)\right) d \mu_{y}\right| \\
& \leq|\lambda| \sup _{x \in M_{b}}\left\{\left|\int_{M_{x} \backslash M_{a}} K(x, y)\left(g_{1}(y)-g_{2}(y)\right) d \mu_{y}\right|\right. \\
& \left.+\left|\int_{M_{x} \cap M_{a}} K(x, y)\left(g_{1}(y)-g_{2}(y)\right) d \mu_{y}\right|\right\} \\
& \leq|\lambda| A \sup _{x \in M_{b}} \mu\left(M_{x} \backslash M_{a}\right)\left\|g_{1}-g_{2}\right\|_{M_{b}}<\frac{1}{2}\left\|g_{1}-g_{2}\right\|_{M_{b}} \text {. }
\end{aligned}
$$

Therefore the operator $\lambda \mathbb{K}_{b}$ is contractive on the set $T$ (it is easy to see that the set $T$ is closed) and from the Banach fixed point theorem it follows that $\lambda \mathbb{K}_{b} / T$ has exactly one fixed point $g_{0} \in T$ such that

$$
\left(\lambda \mathbb{K}_{b} g_{0}\right)(x)=g_{0}(x), \quad x \in M_{b} .
$$

It is immediately verified that (5) has a zero solution and hence from the uniqueness we get $g_{0}(x) \equiv 0$ for $x \in M_{b}$. On the other hand the uniqueness implies that the restriction of $\varphi(x)$ on $M_{b}$ coincides with $g_{0}(x)$ and therefore $\varphi(x) \equiv 0$ for $x \in M_{b}$, i.e. $b \in H$. Thus we proved that the set $H$ together with all its points $a$ contains a neighbourhood of any of these points as well, i.e. $H$ is open.

Furthermore, taking into account that $\Omega$ is connected, we obtain $H=\Omega$ and hence (5) yields $\varphi(x) \equiv 0$ for $x \in \Omega$, i.e. equation (5) for each $\lambda \in \mathbb{C}$ has only the trivial solution. The Fredholm alternative implies that for each $\lambda \in \mathbb{C}$ equation (1) has an unique solution $\varphi(x) \in C(\Omega)$ for each function $f(x) \in C(\Omega)$.

Remark 3. The spectrum $\sigma(\mathbb{K})$ of the operator $\mathbb{K}$ consists of the point $\lambda=0$ only.

Remark 4. The spectral radius $\tau(\mathbb{K})$ of $\mathbb{K}$ is zero. 
The validity of the above statements follows immediately from theorems 1, 2 and from [6] (Theorems 3,4 of Section VIII.2 and Theorems 1, 2 of Section X.5).

Therefore the solution of (1) is given by the equality

$$
\varphi(x)=\left((\mathbb{I}-\lambda \mathbb{K})^{-1} f\right)(x)=\lambda^{-1}\left(R\left(\lambda^{-1} ; \mathbb{K}\right) f\right)(x),
$$

where the resolvent $R\left(\lambda^{-1} ; \mathbb{K}\right)$ is presented in Neumann series convergent in the operator topology for each $\lambda \in \mathbb{C}$,

$$
R\left(\lambda^{-1} ; \mathbb{K}\right)=\lambda I+\lambda^{2} \mathbb{K}+\lambda^{3} \mathbb{K}^{2}+\cdots
$$

or in a more expanded form

$$
\lambda^{-1}\left(\mathbb{R}\left(\lambda^{-1} ; \mathbb{K}\right) f\right)(x)=f(x)+\lambda \int_{M_{x}} K(x, y) f(y) d \mu_{y}+\cdots
$$

where the series (7) is uniformly convergent for each $\lambda \in \mathbb{C}$ ([6] Theorem 3 of Section VII.2).

In particular if $f(x) \equiv 1$ and $\lambda=1$ we obtain from (7) a special solution $\phi(x)$ of equation (1):

(8) $\phi(x)=1+\int_{M_{x}} K(x, y) d \mu_{y}+\int_{M_{x}} K(x, y)\left(\int_{M_{y}} K\left(y, y_{1}\right) d \mu_{y_{1}}\right) d \mu_{y}+\cdots$

THEOREM 3. Let conditions $(A)$ be fulfilled and let the continuous function $\psi: \Omega \rightarrow \mathbb{R}$ satisfy for each $x \in \Omega$ the inequality

$$
\psi(x) \leq f(x)+\int_{M_{x}} K(x, y) \psi(y) d \mu_{y},
$$

where the functions $f: \Omega \rightarrow \mathbb{R}, K: \Omega^{2} \rightarrow \mathbb{R}$ are continuous, and $K(x, y) \geqslant 0$ for $x, y \in \Omega$. Then if we denote by $\varphi(x)$ the solution of the equation

$$
\varphi(x)=f(x)+\int_{M_{x}} K(x, y) \varphi(y) d \mu_{y},
$$

then for each $x \in \Omega$ the inequality

$$
\psi(x) \leq \varphi(x)
$$

holds.

Proof. Iterating $n$ times the right hand side of (9) we obtain

$$
\begin{gathered}
\text { (10) } \psi(x) \leq f(x)+\int_{M_{x}} K(x, y) f(y) d \mu_{y}+\int_{M_{x}} K(x, y)\left(\int_{M_{y}} K\left(y, y_{1}\right) f\left(y_{1}\right) d \mu_{y_{1}}\right) d \mu_{y} \\
+\cdots+\int_{M_{x}} K(x, y) \cdots\left(\int_{M_{y_{n}}} K\left(y_{n-1}, y_{n}\right) \psi\left(y_{n}\right) d \mu_{y_{n}}\right) \cdots d \mu_{y} .
\end{gathered}
$$


Taking into account (6) and (7) and passing to the limit $n \rightarrow+\infty$ in (10) we obtain

$$
\psi(x) \leq f(x)+\int_{M_{x}} K(x, y) f(y) d \mu_{y}+\cdots=\varphi(x) .
$$

Theorems 2 and 3 can be naturally applied to the case when $\Omega$ is not compact or connected but the mapping $M$ satisfies certain additional conditions. For example, it is sufficient to require that every nonempty set $M_{x}$, $x \in \Omega$, should be compact, connected, have a finite measure (assuming that $\mu$ takes the value $+\infty$ as well) and, besides, should contain a point $y=y(x)$ for which $\mu\left(M_{y}\right)=0$. In that case all considerations about the operator $\mathbb{R}$ must be referred to its restriction $\mathbb{K}_{a}$, where $a \in \Omega$ is an arbitrary point.

Let us consider in particular the inequality

$$
\psi(x) \leq C+\int_{M_{x}} K(y) \psi(y) d \mu_{y}, \quad x \in \Omega
$$

where $C$ is an arbitrary constant, $K: \Omega \rightarrow \mathbb{R}$ is a continuous function, and $K(y) \geqslant 0, y \in \Omega$. Then Theorem 3 and equality (8) yield

$$
\psi(x) \leq C \phi(x), \quad x \in \Omega
$$

where the function $\phi(x)$ is a solution of the equation

$$
\phi(x)=1+\int_{M_{x}} K(y) \phi(y) d \mu_{y},
$$

and is presented in the form

$$
\phi(x)=1+\int_{M_{x}} K(y) d \mu_{y}+\cdots
$$

where the series (13) is uniformly convergent.

In fact (11) presents an analog of the Bellman-Gronwall inequality.

If we set $K(y) \equiv 1$ in (13) then we have an analog of the exponent corresponding to the mapping $M$ :

$$
\exp _{M}(x)=1+\int_{M_{x}} d \mu_{y}+\int_{M_{x}}\left(\int_{M_{y}} d \mu_{y_{1}}\right) d \mu_{y}+\cdots
$$

To illustrate the results obtained we shall consider a few examples in the case when $\Omega$ is finite dimensional.

Example 1 . Let $\Omega=\mathbb{R}_{+}, \mathbb{R}_{+}=[0,+\infty), M_{x}=[0, x]$. Then $\exp _{M}(x)=e^{x}$ and in inequality (11) we have 


$$
\phi(x)=\exp \left[\int_{0}^{x} K(y) d y\right]
$$

Example 2。 Let $\Omega=\mathbb{R}_{+}, M_{x}=[0, \psi(x)]$, where $\psi(x)$ is continuously differentiable and $0 \leq \psi(x) \leq x$. Then $\exp _{M}(x)$ is a solution of the Cauchy problem

$$
\phi^{\prime}(x)=\psi^{\prime}(x) \phi(\psi(x)), \quad \phi(0)=1
$$

If in particular $\psi(x)=\frac{x}{2}$ then

$$
\exp _{M}(x)=\sum_{n=0}^{+\infty} \frac{x^{n}}{2^{n(n+1) / 2} n !}
$$

Example 3。 Let $\Omega=\mathbb{R}_{+}^{2}, x=\left(x_{1}, x_{2}\right), x_{1}, x_{2} \geqslant 0$, and

$$
M_{x}=\left\{\left(y_{1}, y_{2}\right) \mid 0 \leq y_{1} \leq x_{1}+x_{2}, 0 \leq y_{2} \leq x_{1}+x_{2}-y_{1}\right\} .
$$

Then $\exp _{M}(x)=\cosh \left(x_{1}+x_{2}\right)$.

Example 4 [4]. Let $\Omega=\mathbb{R}_{+}^{n}, x=\left(x_{1}, \cdots, x_{n}\right), x_{i} \geqslant 0, i=\overline{1, n}$ and $M_{x}=$ $\left[0, x_{1}\right] \times \cdots \times\left[0, x_{n}\right]$. Then

$$
\exp _{M}(x)=\sum_{i=1}^{\infty} \frac{\left(x_{1} \cdots x_{n}\right)^{i}}{(i !)^{n}}
$$

The following theorem is valid in the general case:

THEOREM 4. Let $\Omega=\mathbb{R}_{+}^{n}, \mu$ be a Lebesgue measure and let the following conditions be fulfilled:

1. For each $x \in \Omega$ and each $y \in M_{x}$ we have $x_{i} \geqslant y_{i}, i=\overline{1, n}$.

2. The function $K: \Omega \rightarrow \mathbb{R}$ is continuous and $K(y) \geqslant 0$ for $y \in \Omega$.

Then the solution $\phi(x)$ of equation (12) satisfies the inequality

$$
\phi(x) \leq \exp \left[\int_{M_{x}} K(y) d y\right], \quad x \in \Omega
$$

( $A$ similar result in the case when $K(y)$ is an integrable function but the mapping $M$ has a special form is obtained in [7]).

Proof. Let $z \in \mathbb{R}_{+}^{n}$ be a point such that the $n$-dimensional parallelepiped $B_{z}=\left[0, z_{1}\right] \times \cdots \times\left[0, z_{n}\right]$ contains $M_{z}$ and let us choose an arbitrary function $\tilde{K}: B_{z} \rightarrow \mathbb{R}_{+}, \tilde{K} \in C\left(B_{z}\right)$, such that $\tilde{K}(y) \equiv K(y)$ for $y \in M_{z}$. Then the function

$$
\psi(x)=\exp \left[\int_{B_{x}} \tilde{K}(y) d y\right], \quad x \in B_{z}
$$


satisfies the equation

$$
\psi(x)=1+\int_{B_{x}} \mathbb{F}(y) \psi(y) d y,
$$

where $B_{x}=\left[0, x_{1}\right] \times \cdots \times\left[0, x_{n}\right]$, while we shall clear up the form of the function $F$ in the case $n=2$ :

$$
\begin{aligned}
& \psi\left(x_{1}, x_{2}\right)=\exp \left[\int_{0}^{x_{1}}\left(\int_{0}^{x_{2}} \tilde{K}\left(\xi_{1}, \xi_{2}\right) d \xi_{2}\right) d \xi_{1}\right]=1+\int_{0}^{x_{1}}\left(\frac{\partial}{\partial y_{1}} \psi\left(y_{1}, x_{2}\right)\right) d y_{1} \\
& =1+\int_{0}^{x_{1}}\left(\exp \left[\int_{0}^{y_{1}}\left(\int_{0}^{x_{2}} \widetilde{K}\left(\xi_{1}, \xi_{2}\right) d \xi_{2}\right) d \xi_{1}\right] \cdot \int_{0}^{x_{2}} \tilde{K}\left(y_{1}, \xi_{2}\right) d \xi_{2}\right) d y_{1} \\
& =1+\int_{0}^{x_{2}}\left[\frac{\partial}{\partial y_{2}}\left(\int_{0}^{x_{1}}\left(\exp \left[\int_{0}^{y_{1}}\left(\int_{0}^{y_{2}} \tilde{K}\left(\xi_{1}, \xi_{2}\right) d \xi_{2}\right) d \xi_{1}\right] \cdot \int_{0}^{y_{2}} \tilde{\mathbb{K}}\left(y_{1}, \xi_{2}\right) d \xi_{2}\right) d y_{1}\right)\right] d y_{2} \\
& =1+\int_{0}^{x_{2}}\left[\int _ { 0 } ^ { x _ { 1 } } \operatorname { e x p } [ \int _ { 0 } ^ { y _ { 1 } } ( \int _ { 0 } ^ { y _ { 2 } } \widetilde { K } ( \xi _ { 1 } , \xi _ { 2 } ) d \xi _ { 2 } ) d \xi _ { 1 } ] \left(\int_{0}^{y_{1}} \tilde{K}\left(\xi_{1}, y_{2}\right) d \xi_{1} \circ \int_{0}^{y_{2}} \widetilde{K}\left(y_{1}, \xi_{2}\right) d \xi_{2}\right.\right. \\
& \left.\left.\left.\quad+\widetilde{K}\left(y_{1}, y_{2}\right)\right) d y_{1}\right)\right] d y_{2},
\end{aligned}
$$

i.e.

$$
F\left(x_{1}, x_{2}\right)=\int_{0}^{x_{1}} \tilde{K}\left(y_{1}, x_{2}\right) d y_{1}^{\circ} \int_{0}^{x_{2}} \tilde{K}\left(x_{1}, y_{2}\right) d y_{2}+\tilde{K}\left(x_{1}, x_{2}\right) .
$$

It is not difficult to verify that for an arbitrary $n$ the function $\mathbb{F}$ is equal to the sum of $\widetilde{K}$ and a polynomial with positive coefficients of integrals of $\widetilde{K}$ with multiplicity from 1 to $n-1$ and with integration bounds from 0 to $x_{i}$. Hence $F(x) \geqslant \tilde{K}(x)$ for $x \in B_{z}$ and from equation (14) we obtain

$$
\psi(x) \geqslant 1+\int_{M_{x}} \tilde{K}(y) \psi(y) d y=1+\int_{M_{x}} \mathbb{K}(y) \psi(y) d y .
$$

Therefore from Theorem 3 taking into account that $\phi(x)$ is a solution of (12) we obtain the inequality $\phi(x) \leq \psi(x)$ which had to be proved.

\section{References}

[1] E.F. Beckenbach and R. Bellman, Inequaliiies, Springer-Verlag, Berlin, 1965.

[2] V. Lakshmikantham and S. Leela, Differential and Integral Inequalities, 1. Academic Press, New York, 1969.

[3] A.I. Zahariev and D.D. Bainov, A note on Bellman-Gronwall's inequality, J. Math. Anal. Appl., $\mathbb{1}$ (1981), 147-149.

[4] W. Walter, Differential and Integral Inequalities, Springer-Verlag, Berlin, 1970.

[5] Functional Analysis, Series "Niathematical Reference Library", Izdat. Nauka, Moscow, 1972 (in Russian).

[6] K. Yosida, Functional Analysis, Springer-Verlag, Berlin, 1965.

[7] A.M. Fink, Wendoff's inequalities, NATMA, 5 (1981), 873-875. 
